\title{
REFERENCES
} 297-310.

1. B. R. Gelbaum, Tensor products of Banach algebras, Canad. J. Math. 11 (1959),

2. - Note on the tensor product of Banach algebras, Proc. Amer. Math. Soc. 12 (1961), 750-757.

3. S. Mac Lane, Homology, Springer-Verlag, Berlin, 1963.

4. C. E. Rickart, Banach algebras, Van Nostrand, New York, 1960.

University of California, Irvine

\section{ON EIGENVALUE DISTRIBUTIONS FOR ELLIPTIC OPERATORS WITHOUT SMOOTH COEFFICIENTS. II}

\author{
BY RICHARD BEALS ${ }^{1}$
}

Communicated by Gian-Carlo Rota, April 5, 1968

In two previous papers the known asymptotic formula for the eigenvalues of a selfadjoint elliptic boundary value problem was extended to some cases of operators without smooth coefficients: to the Dirichlet problem in [1] and to the general coercive differential boundary value problem in [2]. The object of this note is to complete this study by proving the formula for general (i.e. not necessarily differential) boundary value problems on domains without smooth boundary. We use the methods of [1], [2]. It should be noted that the case of differential boundary conditions can be handled in a different way; see [3].

Let $\Omega$ be a bounded open set in $R^{n}$ with boundary $\partial \Omega$ which is regular in the sense of Calderón [4], i.e. satisfying the "restricted cone condition." Let $\boldsymbol{A}=\sum a_{\alpha}(x) D^{\alpha}$ be an operator defined on $\Omega$, with coefficients in $L^{\infty}(\Omega)$ and top-order coefficients uniformly continuous on $\Omega$. We assume that $\boldsymbol{A}$ is formally selfadjoint and uniformly elliptic of order $m$. Let $A$ be a selfadjoint realization of $\boldsymbol{A}$ in $L^{2}(\Omega)$, with domain $D(A) \subset H^{m}(\Omega)$. Set

$$
c(A)=(2 \pi)^{-n} \int_{\Omega} \int_{a(x, \xi)<1} d \xi d x,
$$

\footnotetext{
${ }^{1}$ Research partially supported by a contract with the Army Research OfficeDurham.
} 
where $a(x, \xi)$ is the characteristic polynomial of $\boldsymbol{A}$ at $x$.

Theorem. For $\lambda>0$, let $N(\lambda)$ be the number of eigenvalues of $A$ with absolute value $<\lambda$, counting multiplicity. Then

$$
N(\lambda) \sim c(A) \lambda^{n / m} \quad \text { as } \lambda \rightarrow \infty .
$$

We may replace $A$ by $A+t I$ for some real $t$ and assume that $A$ has a bounded inverse $S$. Set

$$
\begin{gathered}
\mu_{j}(S)=\inf _{\operatorname{codim} K=j-1} \sup _{u \in K,\|u\|=1}\|S u\|, \\
\alpha(S)=\liminf _{j \rightarrow \infty} j^{-m / n} \mu_{j}(S), \\
\beta(S)=\limsup _{j \rightarrow \infty} j^{-m / n} \mu_{j}(S) .
\end{gathered}
$$

The assertion of the Theorem is equivalent to $\alpha(S)=\beta(S)=d(\boldsymbol{A})$, where

$$
d(\boldsymbol{A})=(c(\boldsymbol{A}))^{m / n} .
$$

By Theorem 5.2 of $[2], \alpha(S) \geqq d(\boldsymbol{A})$. We must show $\beta(S) \leqq d(\boldsymbol{A})$.

Let $\boldsymbol{A}_{\epsilon}, 0<\epsilon<1$, be a family of formally selfadjoint operators of order $m$ defined on a neighborhood of $\Omega \cup \partial \Omega$ and having $C^{\infty}$ coefficients, such that the coefficients of the principal parts converge uniformly on $\Omega$ to those of the principal part of $A$ as $\epsilon \rightarrow 0$. Let $A_{\epsilon}$ be the restriction of $\boldsymbol{A}_{\boldsymbol{\epsilon}}$ to $D(A)$. For small $\epsilon, \boldsymbol{A}_{\epsilon}$ is uniformly elliptic on $\Omega$ and $A_{\boldsymbol{\epsilon}}$ has a bounded inverse $S_{\epsilon}$. By Theorems 1.5 and 2.3 of [2], $\beta\left(S_{\epsilon}\right) \rightarrow \beta(S)$ as $\epsilon \rightarrow 0$. Clearly $d\left(\boldsymbol{A}_{\epsilon}\right) \rightarrow d(\boldsymbol{A})$, so it suffices to prove the following.

Lемма. Let $\boldsymbol{A}_{0}$ be a formally selfadjoint operator defined on a neighborhood of $\Omega \cup \partial \Omega$, having $C^{\infty}$ coefficients, and uniformly elliptic on $\Omega$, of order $m$. Let $\boldsymbol{A}_{0}$ be the restriction of $\boldsymbol{A}_{0}$ to a domain $D\left(A_{0}\right) \subset H^{m}(\Omega)$. Suppose $A_{0}$ has a bounded inverse $S_{0}$. Then $\beta\left(S_{0}\right) \leqq d\left(A_{0}\right)$.

Proof. Let $\Omega_{1}$ be a bounded neighborhood of $\Omega \cup \partial \Omega$ having a smooth boundary, such that $\boldsymbol{A}_{0}$ is defined and uniformly elliptic on $\Omega_{1}$. Let $A_{1}$ be the Dirichlet realization of $A_{0}$ in $L^{2}\left(\Omega_{1}\right)$. Then $A_{1}$ is selfadjoint and (2) holds for the eigenvalues of $A_{1}$. We may assume $A_{1}$ has a bounded inverse $S_{1}$.

Let $R: L^{2}\left(\Omega_{1}\right) \rightarrow L^{2}(\Omega)$ be the restriction mapping and $J=R^{*}: L^{2}(\Omega)$ $\rightarrow L^{2}\left(\Omega_{1}\right)$ the extension mapping. Then $J R=P$, the projection of $L^{2}\left(\Omega_{1}\right)$ on to functions vanishing outside $\Omega$. Let $H_{1}=\left\{u \mid R S_{1} u \in D\left(A_{0}\right)\right\}$. 
This is a closed subspace of $L^{2}\left(\Omega_{1}\right)$; let $P_{1}$ be the corresponding projection. Then $S_{0} R P_{1}=R S_{1} P_{1}$. If $v \in D\left(A_{0}\right) \subset H^{m}(\Omega)$, it can be extended [4] to a $u \in H_{c}^{m}\left(\Omega_{1}\right) \subset D\left(A_{1}\right)$. Therefore $R$ maps $H_{1}$ onto $L^{2}(\Omega)$. Then $J$ is a unitary mapping of $L^{2}(\Omega)$ onto $P H_{1}=P L^{2}\left(\Omega_{1}\right)$ and $R$ is its inverse; $S_{0}$ corresponds to $J S_{0} R$ acting on $P H_{1}=P P_{1} L^{2}\left(\Omega_{1}\right)$. Therefore

$$
\begin{aligned}
\mu_{j}\left(S_{0}\right) & \leqq \mu_{j}\left(J S_{0} R P P_{1}\right)=\mu_{j}\left(J S_{0} R P_{1}\right) \\
& =\mu_{j}\left(J R S_{1} P_{1}\right)=\mu_{j}\left(P S_{1} P_{1}\right) \leqq \mu_{j}(S),
\end{aligned}
$$

and $\beta\left(S_{0}\right) \leqq \beta\left(S_{1}\right)=d\left(\boldsymbol{A}_{1}\right)$, where $\boldsymbol{A}_{1}$ denotes $\boldsymbol{A}_{0}$ considered as defined on $\Omega_{1}$ rather than on $\Omega$. We get the desired conclusion by shrinking $\Omega_{1}$.

\section{REFERENCES}

1. R. Beals, On eigenvalue distributions for elliptic operators without smooth coefficients, Bull. Amer. Math. Soc. 72 (1966), 701-705.

2. - Classes of compact operators and eigenvalue distributions for elliptic operators, Amer. J. Math. 89 (1967), 1056-1072.

3. - Global asymptotic estimates for elliptic spectral functions and eigenvalues, Bull. Amer. Math. Soc. 74 (1968), 358-360.

4. A. P. Calderon, Lebesgue spaces of differentiable functions and distributions, Proc. Sympos. in Pure Math., vol. 4, Amer. Math. Soc., Providence, R. I., 1961.

University of Chicago 